\title{
PLANETARY NEBULAE WITH BINARY NUCLEI
}

\author{
MARIO LIVIO \\ Space Telescope Science Institute \\ 3700 San Martin Drive, Baltimore, MD 21218 \\ e-mail: mlivio@stsci.edu
}

\begin{abstract}
.
High resolution images of planetary nebulae have revealed a variety of non-spherical morphologies. In addition, some planetary nebulae were found to produce highly collimated jets. It is argued that binary central stars may play a crucial role in the production of all of these morphologies. In particular, a specific mechanism is identified for the generation of "point-symmetric" nebulae. It is shown that the study of binary nuclei of planetary nebulae can provide essential information for the understanding of the common envelope phase in the evolution of binary systems.
\end{abstract}

\section{Introduction}

Planetary nebulae (PNe) exist in a variety of axisymmetric morphologies. These include rings (e.g. Sc We 2; Schwarz, Corradi \& Melnick 1992), bipolar bubbles or hourglasses (e.g. NGC 2346; Bond \& Livio 1990; My Cn 18; Sahai \& Trauger 1996) and highly collimated jets (e.g. NGC 7009; Schwarz et al. 1992; K1-2; Pollacco \& Bell 1996; M1-92; Trammell \& Goodrich 1996). In addition, there exist $\mathrm{PNe}$ which are point-symmetric (e.g. IC 4634; Schwarz et al. 1992; NGC 5307; Bond et al. 1996). In some cases, many of these features appear together (e.g. NGC 6543; Harrington \& Borkowski 1995).

One important clue towards the understanding of the mechanism(s) that is (are) responsible for the formation of the axisymmetric $\mathrm{PNe}$ lies in the fact that many late asymptotic giant branch (AGB) stars, post AGB stars and proto PNe show already a bipolar structure (e.g. Meixner 1993; Kwok 1993; Trammell \& Dinerstein 1994). Examples are: OH 0739-14, HD 44179, IRAS $21282+5050,17150-3224$, and 17441-2411. 


\section{PLANETARY NEBULAE WITH BINARY NUCLEI}

The first important thing to note is the fact that there exists a model which has demonstrated convincingly that it is able to produce a variety of axisymmetric morphologies. This is the "interacting winds" model in the presence of an equatorial to polar density contrast.

\section{The Interacting Winds Model in the Presence of a Density Contrast}

Following the original suggestion by Balick (1987), numerical simulations have demonstrated that when the "interacting winds" model (Kwok 1982; Kahn 1982) is allowed to operate in the presence of a "density contrast" in the slow stellar wind, a bipolar morphology is indeed obtained (Soker \& Livio 1989; Icke, Balick \& Frank 1992; Frank et al. 1993; Mellema 1995). This model works as follows: it is assumed that the slow wind ejected by the star (e.g. the $\sim 20 \mathrm{~km} \mathrm{~s}^{-1}$ wind of an AGB star) contains a non-spherical density distribution, with the material being denser in the equator than in the polar direction. The fast $\left(\sim 1000 \mathrm{~km} \mathrm{~s}^{-1}\right)$, spherically symmetric wind that is emitted later, by the exposed nucleus, catches-up with the slowly moving material and shocks it. Because of the density contrast between the equatorial and polar directions, the fast wind can penetrate more easily at the poles, thus forming an axisymmetric nebula. The works of Frank et al. (1993) and Mellema (1995) have demonstrated then when the nebular inclination angle is taken into consideration, most of the observed morphologies can be reproduced (point-symmetric nebulae and the highly collimated jets observed in some nebulae require additional ingredients, see $\S 4$ ). The main question that therefore arises in the context of this model is: what is the mechanism that is responsible for the formation of a density contrast (in the slow wind) between the equatorial and polar directions?

I should note that an alternative model to "interacting winds" is provided by magnetic shaping (e.g. Pascoli, Leclercq, \& Poulain 1992; Chevalier \& Luo 1994; Garcia-Segura, this conference; and see Livio 1996a for a review). I will not discuss this model here, even though binary central stars can play a role even in the context of this model.

\section{Mechanisms for the Formation of a Density Contrast}

A few mechanisms have been suggested for the formation of the desired density contrast. These include: (i) the inner rim of the protostellar disk (e.g. Balick \& Preston 1988), (ii) an equatorially compressed outflow (e.g. Bjorkman \& Cassinelli 1993), and (iii) the action of a binary companion (e.g. Livio 1994, 1996a; Soker \& Harpaz 1992). Here I will only discuss some aspects of (iii), and I refer the reader to Livio (1996a) for a review of the other possibilities. 


\subsection{COMMON ENVELOPE EVOLUTION}

For relatively close binaries (separation less than about $1000 R_{\odot}$ ), in which the primary can fill its Roche lobe during the AGB phase, an unstable mass transfer is obtained (this is due to the fact that mass is being transferred from the more massive component, which also possesses a deep convective envelope). Thus, a common envelope (CE) phase ensues (see Iben \& Livio 1993 for a comprehensive review), in which, the core of the AGB star and the secondary spiral-in, engulfed by a common, extended envelope. The final outcome of a CE phase can be a substantial reduction in the binary separation (including perhaps a merger in some cases), accompanied by the ejection of the envelope (e.g. Rasio \& Livio 1996). The most detailed common envelope calculation has been recently performed by Rasio \& Livio (1996). This calculation started from an exact hydrostatic equilibrium configuration, at the onset dynamically unstable mass transfer, and was followed by means of a three-dimensional, smooth particle hydrodynamics code, until a quasi-static common envelope configuration was formed (Fig. 1). In the final configuration, it was found that a corotating region of gas was established around the binary. The corotating gas was concentrated to the orbital plane. The subsequent evolution of the system is determined by the viscous coupling between this rigidly rotating core and the outer (differentially rotating) envelope. Multi-dimensional hydrodynamic simulations of the late $\mathrm{CE}$ phase have shown that due to the fact that the spiralling-in binary deposits also angular momentum into the envelope, mass ejection is concentrated to the orbital plane (Livio \& Soker 1988; Taam \& Bodenheimer 1989; Terman, Taam \& Hernquist 1995). Thus, CE evolution generates a density contrast naturally.

\subsection{OBSERVATIONS RELATED TO THE COMMON ENVELOPE PHASE}

There are two classes of objects that we can observe either while in a $\mathrm{CE}$ phase or relatively shortly afterwards. These are, respectively, nova systems and planetary nebulae ( $\mathrm{PNe}$ ) with binary nuclei. In the case of novae, the luminosity of these systems at maximum light $L_{\max } \sim L_{\text {EDDINGTON }} \sim$ $3.8 \times 10^{4} L_{\odot}\left(M / M_{\odot}\right)$ and the effective temperature $T e \lesssim 10^{4} \mathrm{~K}$, imply photospheric radii $R_{\mathrm{ph}} \gtrsim 4 \times 10^{12} \mathrm{~cm}$, well in excess of the binary separations $\left(\sim 1 R_{\odot}\right)$. Thus, the main sequence companion is necessarily engulfed in the expanding nova envelope. I will not discuss nova systems here, a review of the common envelope phase in novae can be found in Livio (1996b).

About $15 \mathrm{PNe}$ with close binary nuclei are now known (Table 1; Bond 1995). Since in order to eject a PN the primary in these systems had to be of an AGB star (or giant) dimensions, the initial separation of the system had to be $\sim 100 R_{\odot}$ or larger. The present separations are of the order of a 


\section{PLANETARY NEBULAE WITH BINARY NUCLEI}
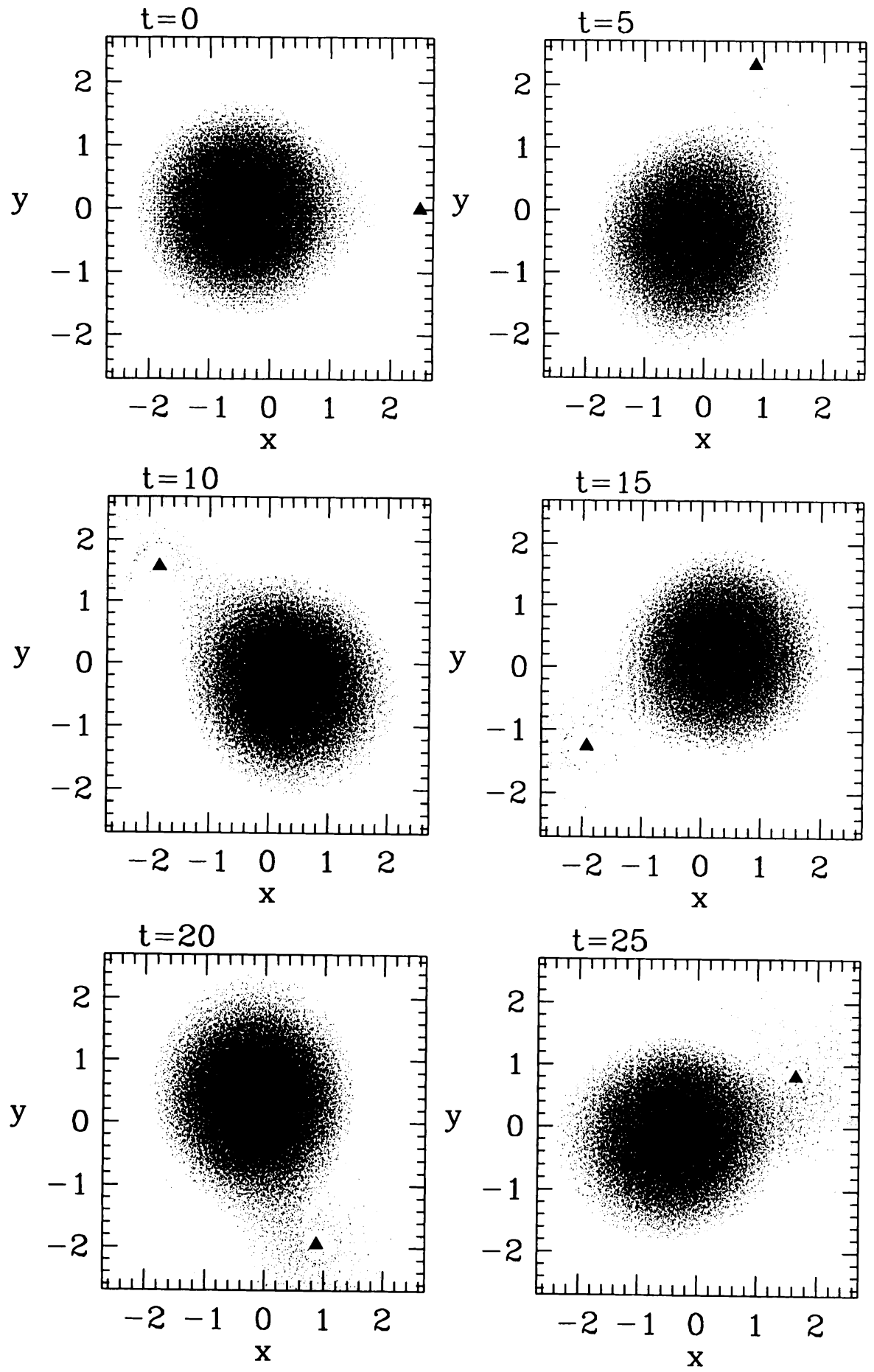

Figure 1. Particle plots showing the evolution of a binary system from the onset of unstable mass transfer to the formation of a common envelope. The giant has a mass of $4 M_{\odot}$ and the secondary of $0.7 M_{\odot}$. From Rasio \& Livio (1996). 


\section{Livio}
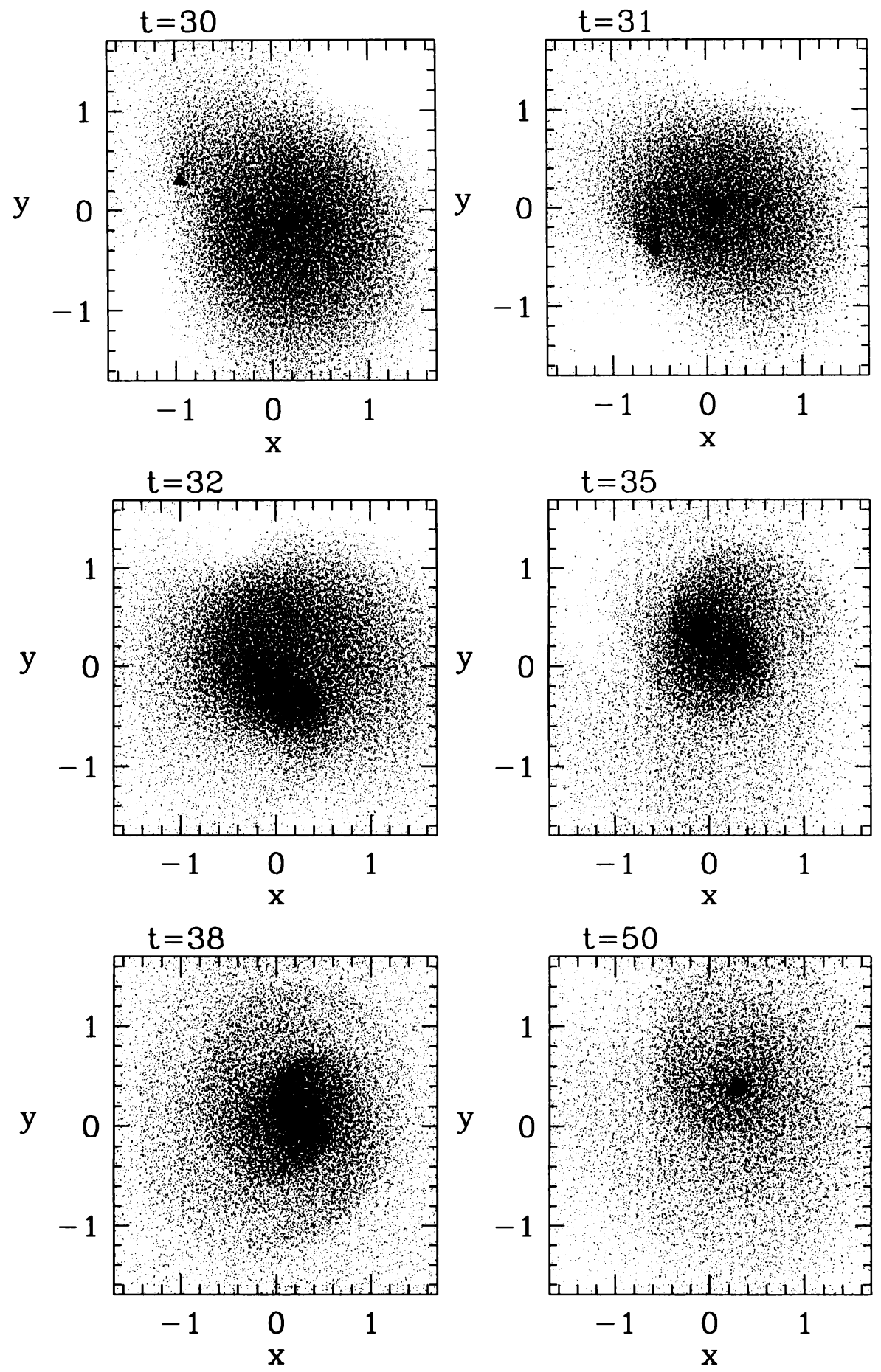

Figure 1. Continued. 
PLANETARY NEBULAE WITH BINARY NUCLEI

Table 1: Planetary Nebulae with Close-Binary Nuclei

\begin{tabular}{lccl}
\hline $\begin{array}{c}\text { Planetary } \\
\text { Nebula }\end{array}$ & $\begin{array}{c}\text { Central } \\
\text { Star }\end{array}$ & $\begin{array}{c}\text { Period } \\
\text { (days) }\end{array}$ & $\begin{array}{c}\text { Binary } \\
\text { Type }\end{array}$ \\
\hline Abell 41 & MT Ser & 0.113 & Reflection \\
DS 1 & KV Vel & 0.357 & Reflection \\
Abell 63 & UU Sge & 0.465 & Eclipsing \\
Abell 46 & V477 Lyr & 0.472 & Eclipsing \\
HFG 1 & V664 Cas & 0.582 & Reflection \\
K 1-2 & VW Pyx & 0.676 & Reflection \\
Abell 65 & $\ldots$ & 1.00 & Reflection \\
HaTr 4 & $\ldots$ & 1.71 & Reflection \\
(Tweedy 1) & BE UMa & 2.29 & Eclipsing \\
Sp 1 & $\ldots$ & 2.91 & Reflection \\
SuWt 2 & $\ldots$ & 4.8 & Eclipsing \\
NGC 2346 & V651 Mon & 15.99 & Spectroscopic \\
Abell 35 & $-22^{\circ} 3467$ & $\ldots$ & IUE composite \\
LoTr 1 & $\ldots$ & $\ldots$ & IUE composite \\
LoTr 5 & HD 112313 & $\ldots$ & IUE composite \\
\hline
\end{tabular}

few $R_{\odot}$, implying that significant shrinkage took place in a CE phase. Since the age of the nebulae is typically less than $\sim 10^{4} \mathrm{yr}$, this means that the observed binaries emerged from the CE less than $10^{4}$ yrs ago.

The question is now how can we use observations of PNe with binary central stars to obtain information on the physics of the CE phase.

\subsection{HOW CAN PLANETARY NEBULAE WITH BINARY NUCLEI BE USED TO CONSTRAIN COMMON ENVELOPE PHYSICS}

The most fundamental parameter in CE evolution is the effeciency parameter $\alpha_{C E}$ (Tutukov \& Yungelson 1979; Livio \& Soker 1988). This is defined as

$$
\alpha_{C E} \equiv \frac{\Delta E_{\text {bind }}}{\Delta E_{\text {orb }}}
$$

where $\Delta E_{\text {bind }}$ is the binding energy of the ejected material, and $\Delta E_{\text {orb }}$ is the change in the orbital energy of the system (between the initial and final configurations). It has not been possible so far to predict the value of $\alpha_{C E}$ theoretically (although the calculations of Rasio \& Livio 1996 do suggest a tentative value of $\alpha_{C E} \sim 1$ ).

There are several ways in which binary PN nuclei can be used (in principle at least) to obtain information on the $\mathrm{CE}$ phase. First, population synthesis calculations can be used, to obtain distributions of the binary 


\section{Livio}

nuclei over the orbital period, the masses of the components, the composition of the nebula etc. Such calculations have been recently performed by de Kool (1990), de Kool \& Ritter (1993) and Yungelson, Tutukov \& Livio (1993). While it is true that population synthesis calculations involve many assumptions (e.g. on the initial distributions over binary parameters, on the evolution of single stars and binary systems, on the value of $\alpha_{C E}$; see de Kool 1996), the results are extremely sensitive to the assumed value of $\alpha_{C E}$. Thus, for example, Yungelson et al. (1993) were able to show that a value of $\alpha_{C E}=10$ (in their definition) is probably inconsistent with the observed period distribution of binary nuclei. A second way in which binary nuclei of $\mathrm{PNe}$ can be used, is by reconstruction of the evolutionary history of individual systems. Examples of this procedure are given in Iben \& Tutukov (1993), Iben \& Livio (1993) and Iben \& Webbink (1989). For example, if we use the fact that there exists a radius-luminosity relation for AGB stars (Eggen \& Iben 1991) of the form $R \simeq 1.0 L^{0.68}$ (where $\mathrm{L}$ and $\mathrm{R}$ are in solar units), together with the assumption that the initial separation satisfies $a_{o} \sim 2 R$. Then approximating eq. (1) (for $M_{1} \gg M_{2}$ ) as $M_{1}^{2} / a_{o} \simeq \alpha_{C E} M_{1 R} M_{2} / a_{f}$ and using the above relations and Kepler's third law give

$$
M_{1} \simeq 0.68 \alpha_{C E}^{1 / 2} L_{10}^{0.34} M_{1 R}^{1 / 2} M_{2}^{1 / 2} P_{o r b}^{-1 / 3}\left(M_{1 R}+M_{2}\right)^{-1 / 6},
$$

where $L_{10}$ is the luminosity (in solar units) of the primary when it filled its Roche lobe, $M_{1 R}$ is the core mass of the primary and $P_{o r b}$ is the orbital period in days. In this way, systems can be placed in the initial-separation primary mass plane (e.g. Iben \& Livio 1993). Conversly, if there exists independent information about the initial parameters $(e . g$. from a cluster age), limits on $\alpha_{C E}$ can be placed. Unfortunately, at the present time, significant uncertainties exist both in the observations of individual systems and in the evolutionary tracks, so that this method gives ambiguous results.

A third way in which $\mathrm{PNe}$ can provide information on the $\mathrm{CE}$ phase is through the morphology of the nebulae. As explained above, the CE phase may be responsible for the generation of the density contrast in the slow wind. In principle therefore, the degree of observed bipolarity can be used together with theoretical modelling, to constrain models for the mass ejection phase during the $\mathrm{CE}$.

Finally, there exists another observational test which can be related directly to the outcome of the CE phase. This has been recently proposed by Sarna et al. (1995). The idea is based on the fact that the ratios of ${ }^{12} C /{ }^{13} C$ and ${ }^{16} \mathrm{O} /{ }^{17} \mathrm{O}$ decrease from values of about 90 and 2700 in stars on the main sequence (respectively; Trimble 1993), to about 13 and 370 in giants and supergiants (Harris, Lambert \& Smith 1988; El Eid 1994). Thus, if during a CE phase which is supposed to lead to a $\mathrm{PN}$ with a binary central star 


\section{PLANETARY NEBULAE WITH BINARY NUCLEI}

(and eventually to a pre-cataclysmic binary), the secondary star accretes some material from the giant's envelope, the ${ }^{12} \mathrm{C} /{ }^{13} \mathrm{C}$ and ${ }^{16} \mathrm{O} /{ }^{17} \mathrm{O}$ ratios in its atmosphere will be different from those of main sequence stars. While the total amount of accreted mass may be rather small (e.g. Hjellming \& Taam 1991) and furthermore, the changes will be diluted by mixing in the secondary's deep convective envelope, one may still expect values that are lower than the main sequence values. This is true in particular for secondaries which are still out of thermal equilibrium and therefore not entirely mixed (e.g. Prialnik \& Livio 1985). Thus, observations of close binary PN nuclei, and in particular those which have oversized secondaries for their masses (e.g. V477 Lyr and UU Sge; Pollacco \& Bell 1994; Bell, Pollacco \& Hilditch 1994), can give important clues on accretion during the CE phase.

\section{Jets and Point-Symmetric Nebulae}

As already noted in the introduction, some $\mathrm{PNe}$ exhibit highly collimated outflows ("jets"; e.g. K1-2). It is not yet clear if such outflows can be the consequence of merely the focussing by oblique shocks, provided by the density contrast (e.g. Icke et al. 1992; Frank, Balick, \& Livio 1996), or if they require the presence of an accretion disk (which is thought to be a necessary ingredient for the formation of the jets observed in Young Stellar Objects and in AGN, e.g. Livio 1996c; a jet emanating from the center of an accretion disk has been observed in $\mathrm{HH} 30$, Burrows et al. 1996). If the latter is true, then for such an accretion disk to form, a binary companion is required, to transfer mass onto the hot AGB star's core. Such a configuration is a possible outcome of the $\mathrm{CE}$ phase, since when the binary emerges from the $\mathrm{CE}$, the secondary star may be in a bloated state (due to the accretion of high entropy material from the CE; see Soker \& Livio 1994; Hjellming \& Taam 1991). Another possibility is that a low mass companion spirals all the way in, and is entirely dissipated to form a disk around the core (Rasio \& Shapiro 1995; Soker 1996; Livio \& Pringle 1996).

It is presently not fully understood how point-symmetric $\mathrm{PNe}$ form. However, it has been suggested by Corradi \& Schwarz (1993) and Livio (1994) that these may represent intermittent ejection episodes of a precessing jet. Three-dimensional numerical hydrodynamic calculations of such a precessing jet, show that point-symmetric configurations that are very similar to the observed $\mathrm{PNe}$, can indeed be obtained (Cliffe et al. 1995). Livio \& Pringle (1996) suggested that the precession of the disk is a consequence of a radiation-induced warp, caused by the irradiation of the disk by the central source. Furthermore, Livio \& Pringle showed that the expected 


\section{Livio}

precession timescale is given by

$\tau_{\text {prec }} \sim 5 \times 10^{3} y r\left(\frac{M_{\text {disk }}}{10^{-3} M_{\odot}}\right)\left(\frac{M_{n u c}}{0.6 M_{\odot}}\right)^{1 / 2}\left(\frac{R_{d i s k}}{2 R_{\odot}}\right)^{-1 / 2}\left(\frac{L_{n u c}}{10^{38} e r g s^{-1}}\right)^{-1}$,

where $M_{n u c}$ and $L_{n u c}$ are the mass and the luminosity of the central source, respectively. Thus, the configuration used in the simulations of Cliffe et al. (1995), can indeed be obtained. This has recently been confirmed by detailed numerical simulations (Pringle \& Livio 1996).

\section{Conclusions}

On the basis of the information presented here, together with discussion in Livio (1996a), the following conservative conclusions can be drawn:

1. Interacting winds, in the presence of a density contrast can produce most of the observed axially-symmetric PN morphologies. It appears that there exists a range of contrasts and/or angular distributions of the densities.

2. Magnetic fields can also produce a range of axially symmetric morphologies.

3. Several mechanisms can produce an equatorial to polar density contrast. Binary central stars provide an attractive option, being able to contribute to most of these mechanisms.

4. Jets are probably emanating from the centers of accretion disks which are threaded by a vertical magnetic field.

5. Point-symmetric nebulae are probably produced by two-sided "wobbling" jets. The accretion disk and the jets are wobbling as a result of a warp induced in the accretion disk by radiation from the central source.

\section{Acknowledgements}

This research has been supported by NASA Grant NAGW-2678 at the Space Telescope Science Institute. 


\section{PLANETARY NEBULAE WITH BINARY NUCLEI}

\section{References}

Balick, B. 1987, A. J., 94, 671.

Balick, B., \& Preston, H. L. 1988, A. J., 94, 958.

Bell, S. A., Pollacco, D. L., \& Hilditch, R. W. 1994, M.N.R.A.S., 270, 449.

Bjorkman, J. E., \& Cassinelli, J. P. 1993, Ap. J., 409, 429.

Bond, H. E. 1995, in Asymmetrical Planetary Nebulae, eds. A. Harpaz, \& N. Soker (Bristol: Institute of Physics Publishing), p. 61.

Bond, H. E., Ciardullo, R., Fullton, L. K., \& Schaefer, K. G. 1996, in preparation.

Burrows, C. J. et al. 1996, Ap. J., in press..

Chevalier, R. A., \& Luo, D. 1994, Ap. J., 421, 225.

Cliffe, J. A., Frank, A., Livio, M., \& Jones, T. W. 1995, Ap. J., 447, L49.

Corradi, R. L. M., \& Schwarz, H. E. 1993, A\&A, 268, 714.

de Kool, M. 1990, Ap. J., 358, 189.

de Kool, M. 1996, in Evolutionary Processes in Binary Stars, eds. R. A. M. J. Wijers, M. B. Davies, \& C. A. Tout (Dordrecht: Kluwer), p. 365.

de Kool, M., \& Ritter, H. 1993, $A \& A, 267,397$.

El Eid, M. F. 1994, $A \& A$, 285, 915.

Frank, A., Balick, B., Icke, V., \& Mellema, G. 1993, Ap. J., 404, L25.

Frank, A., Balick, B., \& Livio, M. 1996, Ap. J., in press..

Harrington, J. P., \& Borkowksi, K. J. 1995, presented at the 185th AAS, Tucson, AZ.

Harris, M. L., Lambert, D. L., \& Smith, V. V. 1988, Ap. J., 325, 768.

Hjellming, M. S., \& Taam, R. E. 1991, Ap. J., 370, 709.

Iben, I., Jr., \& Livio, M. 1993, Pub. A.S.P., 105, 1373.

Iben, I., Jr., \& Tutukov, A. V. 1993, Ap. J., 418, 343.

Iben, I., Jr., \& Webbink, R. F. 1989, in White Dwarfs, ed. G. Wegner (Berlin: SpringerVerlog), p. 477.

Icke, V., Balick, B., Frank, A. 1992, $A \mathscr{E} A, \mathbf{2 5 3}, 224$.

Icke, V., Mellema, G., Balick, B., Eulderink, F., \& Frank, A. 1992, Nature, 355,524.

Kahn, F. D. 1982, in IAU Symp. 103, Planetary Nebulae, ed. D. R. Flower (Dordrecht: Reidel), p. 305.

Kwok, S. 1982, Ap. J., 258, 280.

Kwok, S. 1993, in Luminous High-Latitude Stars, ASP Conf. Series 45, (San Francisco: ASP), p. 348.

Livio, M. 1994, in Circumstellar Media in the Late Stages of Stellar Evolution, eds. R. Clegg et al. (Cambridge: Cambridge University Press), p. 35.

Livio, M. 1996a, in Science with the Hubble Space Telescope-II, eds. P. Benvenuti, F. D. Macchetto, \& E. J. Schreier (Baltimore: ST ScI), p. 257.

Livio, M. 1996b, in Evolutionary Processes in Binary Stars, eds. R. A. M. J. Wijers, M. B. Davies, \& C. A. Tout (Dordrecht: Kluwer), p. 141.

Livio, M. 1996c, in IAU Colloq. 163, Accretion Phenomena \& Related Objects, eds. L. Ferrario et al. , in press.

Livio, M., \& Pringle, J. E. 1996, Ap. J., 465, L55.

Livio, M., \& Soker, N. 1988, Ap. J., 329, 764.

Meixmer, M. 1993, PhD Thesis, University of California, Berkeley.

Mellema, G. 1995, M.N.R.A.S., 277, 173.

Pascoli, G., Leclercq, J., \& Poulain, B. 1992, Pub. A.S.P., 104, 182.

Pollacco, D. L., \& Bell, S. A. 1994, M.N.R.A.S., 267, 452.

Pollacco, D. L., \& Bell, S. A. 1996, private communication.

Prialnik, D., \& Livio, M. 1985, M.N.R.A.S., 216, 37.

Prinlge, J. E., \& Livio, M. 1996, in preparation.

Rasio, F. A., \& Livio, M. 1996, Ap. J., 471, 366.

Sahai, R., \& Trauger, J. T. 1996, HST press release.

Sarna, M. J., Dhillon, V. S., Marsh, T. R., \& Marks, P. B. 1995, M.N.R.A.S., 272, L41.

Schwarz, H. E., Corradi, R. L. M., \& Melnick, J. 1992, $A \& A S$, 96, 23. 


\section{Livio}

Soker, N. 1996, preprint.

Soker, N., \& Harpaz, A. 1992, Pub. A.S.P., 104, 923.

Soker, N., \& Livio, M. 1989, Ap. J., 339, 268.

Soker, N., \& Livio, M. 1994, Ap. J., 421, 219.

Taam, R. E., \& Bodenheimer, P. 1989, Ap. J., 337, 849.

Terman, J. L., Taam., R. E., \& Hernquist, L. 1995, Ap. J., 445, 367.

Trammell, S. R., \& Dinerstein, H. L. 1994, A. J., 108, 984.

Trammell, S. R., \& Goodrich, R. W. 1996, Ap. J., in press..

Trimble, V. 1993, $A \boxminus A R, 3,1$.

Tutukov, A. V., \& Yungelson, L. R. 1979, Acta Astron., 29, 666.

Yungelson, L. R., Tutukov, A. V., \& Livio, M. 1993, Ap. J., 418, 794. 